\title{
Breakthrough in carbon nanotube growth: unique alloy nanocatalysts lead to the chirality specified tubes
}

Since Dr. Iijima reported his observation of tubular carbon under transmission electron microscopy (TEM) in 1991, carbon nanotubes (CNTs) have attracted intensive attention. A single-walled CNT (SWNT) is a seamless cylinder formed by rolling a piece of graphene, the honeycombstructured $\mathrm{sp}^{2}$ carbon. This unique structure endows SWNTs with amazing properties and great potentials in various fields. However, many of the advanced applications (e.g., nanoelectronics and optoelectronics) require SWNTs of identical structure or chirality. Structure/chirality-specific growth, the ultimate goal in SWNT preparation, has been the most challenging topic in the field since the mid-1990s.

The recent breakthrough made by Yan Li and her group at Peking University was published in Nature on June 26, 2014 [1]. Researchers at Hong Kong Polytechnic University, Institute of Physics in CAS, and Shanghai Synchrotron Radiation Facility also contributed to the work. These researchers used a new family of catalysts, tungsten-based bimetallic-alloy nanoparticles of non-cubic symmetry, which, with high melting points, are thus able to maintain their crystal structure during the chemical vapor deposition

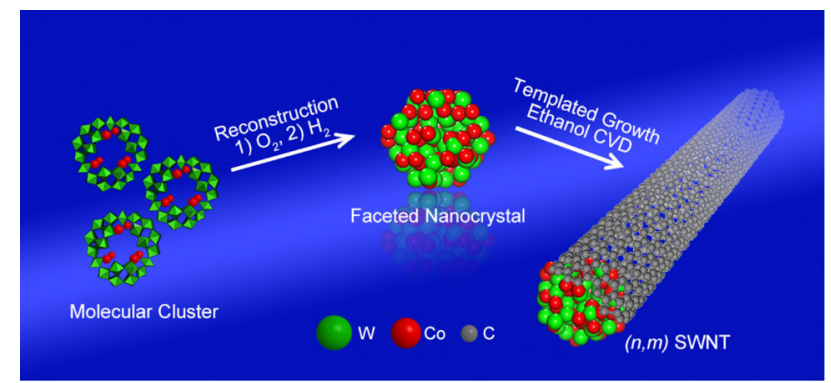

Figure 1 Illustration of the preparation of W-Co nanocrystal and the templated growth of a SWNT with specified $(n, m)$.
(CVD) process, to regulate the chirality of the grown SWNTs. The $(12,6)$ SWNTs are directly synthesized at an abundance of $>92 \%$ with $\mathrm{W}_{6} \mathrm{Co}_{7}$ catalysts (Figure 1). Experimental evidence and theoretical simulation reveal that the good structural match between the carbon atom arrangement around the nanotube circumference and the arrangement of the atoms in one of the planes of the nanocrystal catalyst facilitates the $(n, m)$ preferential growth of SWNTs. This method is also valid for other tungsten-based alloy nanocatalysts to grow SWNTs of various designed chiralities. This work is significant in that it proposed a strategy to realize the structure specified growth of SWNTs, developed a new family of catalysts for SWNT growth, and proposed a method to prepare tungsten-based alloy nanoparticles under moderate conditions with molecular clusters as precursors.

Employing unique-structure alloy nanocrystals as catalysts offers a possible solution for the ultimate goal of selective SWNT growth. This work is expected to promote rapid development in advanced applications of SWNTs. Other research areas might benefit from this work as well.. For example, in alloy metallurgy, choosing different precursor to greatly reduce the alloying temperature may be a strategy to lower energy consumption. A reduced process temperature may then greatly ease both the production apparatus and production control.

LI YaDong

Department of Chemistry, Tsinghua University

1 Yang F, Wang X, Zhang D, Yang J, Luo D, Xu Z, Wei J, Wang JQ, Xu Z, Peng F, Li X, Li R, Li Y, Li M, Bai X, Ding F, Li Y. Chirality-specific growth of single-walled carbon nanotubes on solid alloy catalysts. Nature, 2014, 510: 522-524 